\title{
Scrape-Off Layer Features of the QH-mode
}

C. J. Lasnier, K. H. Burrell, J. S. deGrassie, A. W. Leonard, R. A. Moyer, G. D. Porter, J. G. Watkins,

This article was submitted to $15^{\text {th }}$ International Conference on Plasma Surface Interactions in Controlled Fusion Devices, Gifu, Japan, May 27-31, 2002

\section{May 23, 2002}

U.S. Department of Energy

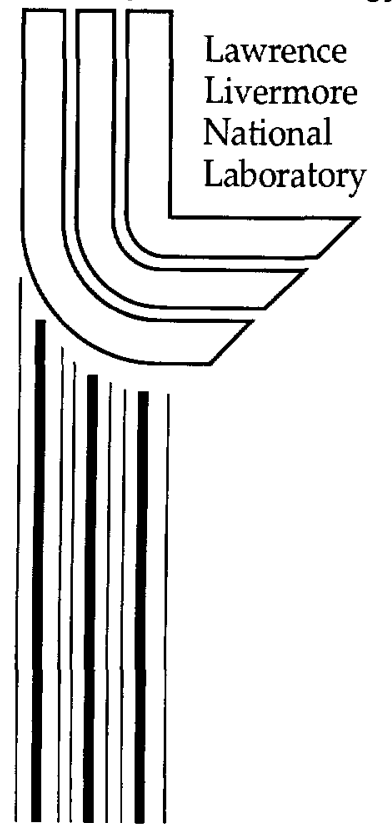




\section{DISCLAIMER}

This document was prepared as an account of work sponsored by an agency of the United States Government. Neither the United States Government nor the University of California nor any of their employees, makes any warranty, express or implied, or assumes any legal liability or responsibility for the accuracy, completeness, or usefulness of any information, apparatus, product, or process disclosed, or represents that its use would not infringe privately owned rights. Reference herein to any specific commercial product, process, or service by trade name, trademark, manufacturer, or otherwise, does not necessarily constitute or imply its endorsement, recommendation, or favoring by the United States Government or the University of California. The views and opinions of authors expressed herein do not necessarily state or reflect those of the United States Government or the University of California, and shall not be used for advertising or product endorsement purposes.

This is a preprint of a paper intended for publication in a journal or proceedings. Since changes may be made before publication, this preprint is made available with the understanding that it will not be cited or reproduced without the permission of the author.

This work was performed under the auspices of the United States Department of Energy by the University of California, Lawrence Livermore National Laboratory under contract No. W-7405-Eng-48.

This report has been reproduced directly from the best available copy.

Available electronically at http://www.doc.gov/bridge

Available for a processing fee to U.S. Department of Energy

And its contractors in paper from

U.S. Department of Energy

Office of Scientific and Technical Information

P.O. Box 62

Oak Ridge, TN 37831-0062

Telephone: (865) 576-8401

Facsimile: (865) 576-5728

E-mail: reports@adonis.osti.gov

Available for the sale to the public from

U.S. Department of Commerce

National Technical Information Service

5285 Port Royal Road

Springfield, VA 22161

Telephone: (800) 553-6847

Facsimile: (703) 605-6900

E-mail: orders@ntis.fedworld.gov

Online ordering: $\underline{\mathrm{http}: / / w w w . n t i s . g o v / o r d e r i n g . h t m}$

\section{OR}

Lawrence Livermore National Laboratory

Technical Information Department's Digital Library

http://www.llnl.gov/tid/Library.html 


\title{
Scrape-Off Layer Features of the QH-mode
}

\author{
C.J. Lasnier, ${ }^{a}$ K.H. Burrell, b J.S. deGrassie, ${ }^{b}$ A.W. Leonard, ${ }^{b}$ R.A. Moyer, ${ }^{c}$ G.D. Porter, ${ }^{a}$ \\ J.G. Watkins, ${ }^{\mathrm{d}}$ and the DIII-D team
${ }^{a}$ Lawrence Livermore National Laboratory, P.O. Box 808, Livermore, California 94551
$b_{\text {General Atomics, P.O. Box 85608, San Diego, California } 92186-5608}$

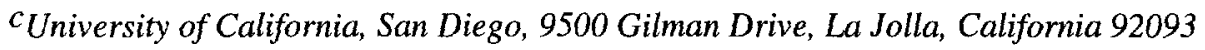 \\ $d_{\text {Sandia National Laboratories, P.O. Box 5800, Albuquerque, New Mexico } 87185}$
}

\begin{abstract}
The quiescent high confinement (QH-mode) and quiescent double barrier (QDB) modes in DIII-D have long-duration H-mode confinement without ELMs, possibly an alternative operating mode in future tokamaks for avoiding damage by ELMs. Instead of ELMs, there is an edge harmonic oscillation (EHO), which is a continuous electromagnetic mode with associated density fluctuations. The edge pedestal is similar to ELMing H-mode, but at very low density to date. We see $\mathrm{C}^{+6}$ ion temperatures of $3-7 \mathrm{keV}$ in scrape-off layer (SOL), $100 \mathrm{kV} / \mathrm{m}$ radial electric fields just inside the separatrix, and a hot area on the divertor baffle whose heating correlates with the presence of the EHO. We attribute the baffle heating to perturbation of trapped ion orbits by the EHO, allowing particles to strike the baffle. The outboard scrape-off layer is wider than the inboard, probably due to lack of trapped ions on the inside.
\end{abstract}

Keywords: SOL, divertor, QH, QDB, EHO, ELM, IRTV, heat flux 


\section{Description of QH- and QDB-modes}

Future large high-power tokamaks may operate in ELMing $\mathrm{H}$-mode, but need to avoid ELMs energetic enough to damage the plasma facing components in the divertors [1,2]. It would be desirable to operate such a device in a regime having confinement characteristics equivalent to $\mathrm{H}$-mode but without ELMs.

In this paper, we examine the scrape-off layer (SOL) plasma of the quiescent highconfinement mode ( $\mathrm{QH}$-mode) in DIII-D. The $\mathrm{QH}$-mode is a regime of long-duration stationary H-mode performance without ELMs [3-5], so far observed only at low density with counter neutral beam injection (NBI), strong pumping, and a large outer gap of $10 \mathrm{~cm}$ or more between the separatrix and the wall. $\mathrm{QH}$-mode has been sustained until neutral beams were shut off, for $3.5 \mathrm{~s}$ or about 25 energy confinement times. The edge profiles of the $\mathrm{QH}$ plasma strongly resemble ELMing $\mathrm{H}$-mode profiles. The edge density pedestal is low $\left(\sim 0.2 \mathrm{n}_{\mathrm{GW}}\right.$, where is $\mathrm{n}_{\mathrm{GW}}$ the Greenwald density) due to strong pumping [3].

In place of ELMs, there is an edge MHD mode that ejects particles and allows pumping for density control. This is usually the edge harmonic oscillation (EHO), a steadily oscillating (not bursting) mode near the separatrix. The EHO prevents the uncontrolled density increase found in standard ELM-free H-mode.

The QDB adds to the QH profiles an inner transport barrier at $\rho \sim 0.4-0.5$ [3]. Most QH-modes in DIII-D contain this inner barrier. In the QDB-mode, we see peaked density profiles and an accumulation of nickel and copper impurities in the core. The density peaking and impurity accumulation are much weaker in $\mathrm{QH}$-mode without a strong inner transport barrier $\left(Z_{\mathrm{eff}}=3\right.$ rather than $\left.\mathrm{Z}_{\mathrm{eff}}=6\right)[6]$.

Magnetic sensors show that the EHO has toroidal mode numbers of $n=1$ up to $n=7$ simultaneously. The fundamental frequency is often near $6 \mathrm{kHz}$ but varies with plasma conditions. The edge pedestal electron density and temperature are hardly changed by the transition to QH-mode. 
The QH-mode occurred with ion $\nabla \mathrm{B}$ drift toward and away from the divertor, and in both lower and upper single-null. All the data shown in this paper are for counter NBI, upper singlenull diverted discharges with ion $\nabla \mathrm{B}$ drift away from the divertor, where most of the DIII-D EHO and QH-mode operation has occurred to date. Detailed descriptions of the quiescent $\mathrm{H}-$ mode $(\mathrm{QH})$ and quiescent double-barrier (QDB) mode are found in Refs. [3-5].

\section{Scrape-off layer features}

The EHO is detectable by most edge diagnostics with high enough time resolution and sampling rate. It has been seen on fixed and reciprocating Langmuir probes, reflectometry, beam emission spectroscopy, millimeter wave scattering, phase contrast imaging, $\mathrm{D}_{\alpha}$ emission, and magnetics. Several of these diagnostics show the associated density oscillation is strongly peaked near the separatrix. Infrared thermography (IRTV) shows a wide distribution of deposited heat flux $\left(q_{d i v}\right)$ in the divertor and on the baffle structure (Fig. 1). This heat flux extends to areas that connect via flux surfaces to the outer midplane more than $6 \mathrm{~cm}$ from the separatrix.

In the $q_{d i v}$ profiles on the flat part of the upper outer baffle ("shelf"), we find an extra peak, 4-5 $\mathrm{cm}$ outside the separatrix when mapped to the outer midplane $\left(\Psi_{\mathrm{N}} \sim 1.04\right)$. This peak is clearly distinct from the outer strike point heat flux peak. The anomalous $q_{d i v}$ peak becomes significant only after the EHO begins.

A divertor Langmuir probe shows particle flux at the same radial location as the anomalous $q_{d i v}$ peak, including ions more energetic than the $200 \mathrm{~V}$ maximum probe bias. If the particles are assumed to have an average energy of $5 \mathrm{keV}$, which is consistent with the ion energy measured by charge exchange recombination spectroscopy (CER), the resulting heat flux is within a factor of two of the thermographic heat flux. This shows the peak is not a reflection or other camera artifact, and proves the presence of charged particle flux.

The CER shows a 3-7 keV population of $\mathrm{C}^{+6}$ ions extending deep into the SOL (Fig. 2), as well as an edge pedestal $\mathrm{C}^{+6}$ temperature of $\sim 5 \mathrm{keV}$. The line emission from these $\mathrm{SOL}$ ions is 
Maxwellian, indicating a thermal population. These hot ions are present in the ELMing phase of the discharge and persist during the EHO. We deduce a hot population of deuterium ions to account for the particle flux measured by the Langmuir probe. We believe it is these $\mathrm{D}$ ions heating the divertor shelf, probably because trapped ion orbits are perturbed by the EHO (see Section 3).

There is an apparent dip in the ion temperature just outside the separatrix, probably due to a cold ion population there, which overwhelms the emission of the hot ions. SOL electrons have temperatures of less than $200 \mathrm{eV}$ with a radial density profile nearly flat at $3-4 \times 10^{18} \mathrm{~m}^{-3}$.

A large radial electric field is detected by both CER (from plasma rotation) and the reciprocating Langmuir probe. The CER shows a large negative field localized just inside the separatrix, roughly five times larger than the corresponding field for ELM-free H-mode (Fig. 2). The reciprocating probe shows a large positive electric field in the deep SOL and a zero crossing in the near SOL. These electric fields will have a strong effect on the trapped ion orbits mentioned above.

There is a much larger $q_{d i}$, peak at the inner strike point $(\mathrm{R}=105 \mathrm{~cm})$. The sharpness of this peak shows that the inboard SOL is narrower than the outboard. If the outboard scrape-off layer is being broadened by the action of the EHO, it may be that the effect of the EHO is strongest at the outside, as has been suggested for ELMs [7] although with a different mechanism. The magnetic measurements do not show a significant decrease in amplitude of the EHO on the inboard side. However, there are few trapped particles on the inboard side to interact with the mode. This difference in the trapped population provides a mechanism for affecting primarily the outboard SOL.

Substantial heat flux also appears at $\mathrm{R} \sim 155 \mathrm{~cm}$ where the flux surfaces are nearly tangential to the sloping baffle surface. If a parallel heat flux $q_{\|}$is calculated, it is more than an order of magnitude larger than on surrounding flux surfaces. However, a divertor Langmuir probe at this location sees very little particle flux, so this heating cannot be due to charged particles. A 
bolometer inversion reveals a concentrated source of radiation in the nearby SOL, which would contribute to heating of this surface (Fig. 3).

Some of the signal detected by bolometers is ascribed to neutral particles striking the detector, demonstrated by signal differences between detectors that view directly and those viewing through the main plasma. The direct view measures higher power, due to neutrals striking the detectors. The inverted 2-D power profile shown in Fig. 3 does not consider neutral power, but the effect is small for this discharge. For other discharges, the effect can be large enough to prevent an acceptable inversion. From this we conclude that neutral particles can carry significant heat to the baffle, although the amount is difficult to quantify.

\section{Ion orbit calculations}

Radiant heating does not adequately account for the heat flux peak seen near $R=170$ on the shelf, particularly since a charged particle flux is measured there. Fixed Langmuir probe data suggests hot ions present in the scrape-off layer, perhaps banana-trapped, flow to the divertor only during the EHO (or in one case tearing modes instead of EHO). However, the particle flux at the location of the anomalous heat flux peak is only weakly modulated at the EHO frequency. There is a strong modulation at $800 \mathrm{~Hz}$ not seen near the separatrix.

Fast ions in the periphery are clearly present in the QH-mode of operation. Counter NBI results in promptly lost orbits for those beam particles ionized within $20-30 \mathrm{~cm}$ of the outboard separatrix. Additionally, the high measured ion temperature and low density in the edge indicates that fast thermal ions can execute collisionless trapped orbits. In Fig. 4, we show the guiding center orbit of an ion for each of these classes. The $80 \mathrm{keV} \mathrm{D}$ ion is representative of one counter injected from an often-used DIII-D beam source. It is launched $6.5 \mathrm{~cm}$ inside the last closed flux surface (LCFS) on the midplane at the pitch angle of the injected neutral and initially will move downward for DIII-D topology. This beam ion then strikes the shelf on the first swing outward (the full orbit is shown as if the boundary did not stop it). This class of ions will clearly deliver 
heat to the upper baffle near $\mathrm{R}=170 \mathrm{~cm}$ but since this heating feature correlates with the EHO we suspect that these beam ions orbits are not the cause of the increased baffle heating during the EHO. Full computations are yet to be done on prompt beam loss to the baffle, taking into account the vertical extent of the NB source.

In the latest set of experiments, we found that heating of this area did not depend strongly on the outer gap, which was varied from more than $10 \mathrm{~cm}$, down to $6 \mathrm{~cm}$ in a discharge. This would have been expected to scrape off some of the trapped beam ions at the smallest gap (Fig. 4). There was no correlation found with the use of a particular beam, nor with the use of varying proportions of "left" and "right" beams, which intersect the plasma tangentially at different major radii. Left beams cause much greater ion orbit losses. There is no evidence of a correlation with direct beam ion losses.

Also shown in Fig. 4 is a $5 \mathrm{keV}$ D ion started $1 \mathrm{~cm}$ beyond the LCFS and with the banana tip just contacting the baffle. The interesting feature of this orbit is that the banana precession frequency is $1.2 \mathrm{kHz}$, very similar to the modulation frequency of the particle flux mentioned above. It is possible that these orbits are being bunched toroidally, perhaps by interaction with the EHO, and thus giving rise to a modulation in the lost orbit flux at the precession frequency. Indeed, the bounce frequency of this orbit is $f_{b} \sim 6.3 \mathrm{kHz}$, which is not too different from the fundamental EHO frequency. We are in the process of doing extensive orbit surveys including the edge electric field, which modifies the toroidal precession velocity of the thermal ions, and seeing if there can be resonances between $f_{b}$ and the EHO frequency, or the harmonics.

\section{Summary and conclusions}

The QH and QDB exhibit H-mode confinement for a duration of many energy confinement times without ELMs and therefore without divertor heat pulses. This is a desirable characteristic for an operating mode in future large tokamaks, in which energetic ELM heat pulses would damage the divertor surfaces. The QH- and QDB-modes have an H-mode edge, and the ELMs 
are replaced by an edge harmonic oscillation. The EHO serves a function similar to ELMs in that it continuously ejects particles from the separatrix, which allows the plasma to be pumped effectively. This is unlike transient standard ELM-free modes, which exhibit monotonically increasing density due to uncontrolled particle confinement, then suffer a large ELM, and finally revert to standard ELMing $\mathrm{H}-$ mode.

We see a $q_{d i v}$ peak on the shelf that we ascribe to fast ions hitting the surface. A diffuse population of 3-7 keV ions is found in the scrape-off layer, present in the ELMing phase of the discharges and remaining in the $\mathrm{QH}$ phase. The measurements by CER are of carbon ions, but we deduce a population of hot deuterium ions. The measured carbon ions appear to be a Maxwellian population but the density is too low to be collisional. This is born out by the low temperatures measured for electrons in the SOL.

From the global behavior, we know that the EHO throws particles into the scrape-off layer, which allows pumping for density control. This is similar to the time-averaged behavior of ELMs. We propose that the EHO also acts on trapped particles near the separatrix, to put some of them on orbits that collide with the divertor baffle.

Both CER and the reciprocating Langmuir probe show much larger $\mathrm{E}_{\mathrm{R}}$ in the boundary than in other confinement modes. The large $\mathrm{E}_{\mathrm{R}}$ will be important in determining the trapped particle orbits.

Beam ion orbit losses may contribute to baffle heating, but only a weak effect is seen from outer gap sweeps. No effect is measured from changing to different beam locations, or a different mix of left and right beams. We believe it is more likely the baffle heating is due to a class of lower-energy trapped thermal ions, whose orbits are perturbed by the EHO enough to hit the baffle. We plan more detailed orbit calculations including the effect of electric fields.

There is a localized high $q_{d i}$ at the inner strike point. If the EHO is playing a role in the broadening of the outer SOL, this indication of a narrow SOL could indicate that the EHO is primarily effective on the outboard side. This is similar to behavior ascribed to ELMs, but for the EHO it could be caused by a lack of trapped particles on the inside. 
If the trapped ion orbits are important in producing the EHO and QH-mode, it is likely to be collisionality of the edge that is important rather than density. If the collisionality is too high, the banana orbits or bunching mechanism could be disrupted. At higher powers and temperatures, the QH-mode may appear at higher densities. There may not be sufficient input power to test this in present devices.

\section{Acknowledgment}

Work supported by U.S. Department of Energy under Contracts W-7405-ENG-48, DEAC03-99ER54463, DE-AC04-94AL85000, and Grant DE-FG03-95ER54294. 


\section{References}

[1] ITER Physics Basis Document, Nucl Fusion 39, (1999) 2137.

[2] A.W. Leonard, A. Hermann, K. Itami, J. Lingertat, A. Loarte, T.H. Osborne, and W. Suttrop, J. Nucl. Mater. 266-269, (1999) 100.

[3] C.M. Greenfield, et al., Phys. Rev. Lett. 86, (2001) 4544.

[4] K.H. Burrell, Phys. Plasmas 8, (2001) 2153.

[5] E.J. Doyle, et al, "The quiescent double barrier regime in the DIII-D tokamak", Proc. of the 28th EPS Conf. on Controlled Fusion and Plasma Physics, Madeira, Portugal, 2001 (European Physical Society) to be published.

[6] W.P. West, private communication (2002).

[7] P.B. Snyder, et al., Phys. Plasmas 9 (2002), 2037. 


\section{Figure Captions}

Fig. 1. (a) Upper divertor Heat flux vs.major radius. The peak in the private flux area is a reflection of the outer strike point. The profile at $1.5 \mathrm{~s}$ is during the ELMing phase; $3 \mathrm{~s}$ is during the EHO. (b) The same heat flux profile overlaid on the baffle structure.

Fig. 2. (a) Ion temperature profile for $\mathrm{C}^{+6}$. The ions in the SOL are $3-7 \mathrm{keV}$. (b) $\mathrm{E}_{\mathrm{R}}$ from CER. There is a large negative electric field just inside the separatrix. (c) $E_{R}$ from reciprocating Langmuir probe. The probe shows a large positive electric field in the far SOL and a zero crossing further in.

Fig. 3. Bolometer inversion, showing a 2-D profile of radiated power.

Fig. 4. Trapped ion orbits for $80 \mathrm{keV}$ and $5 \mathrm{keV}$ deuterium ions. 

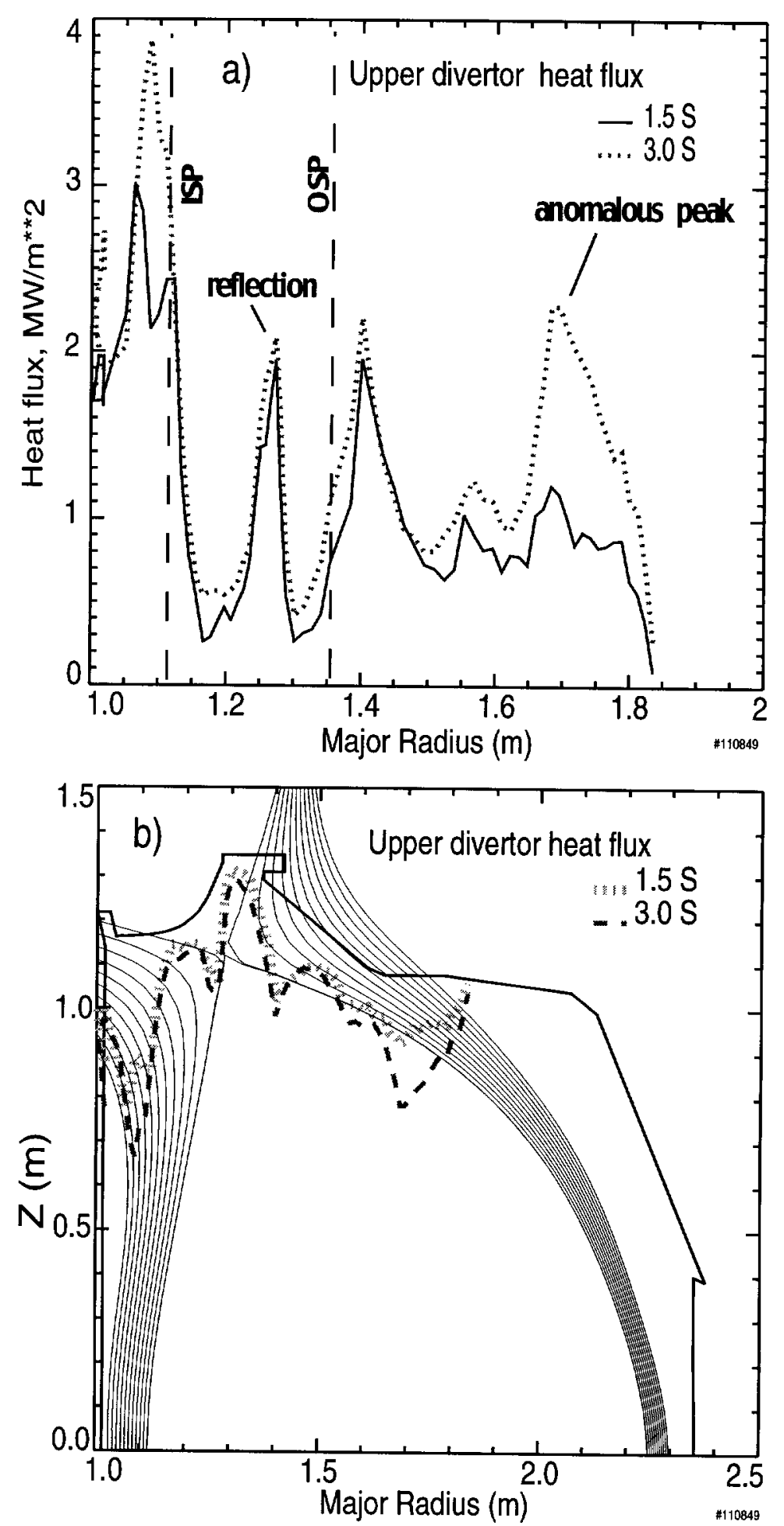

Figure 1 

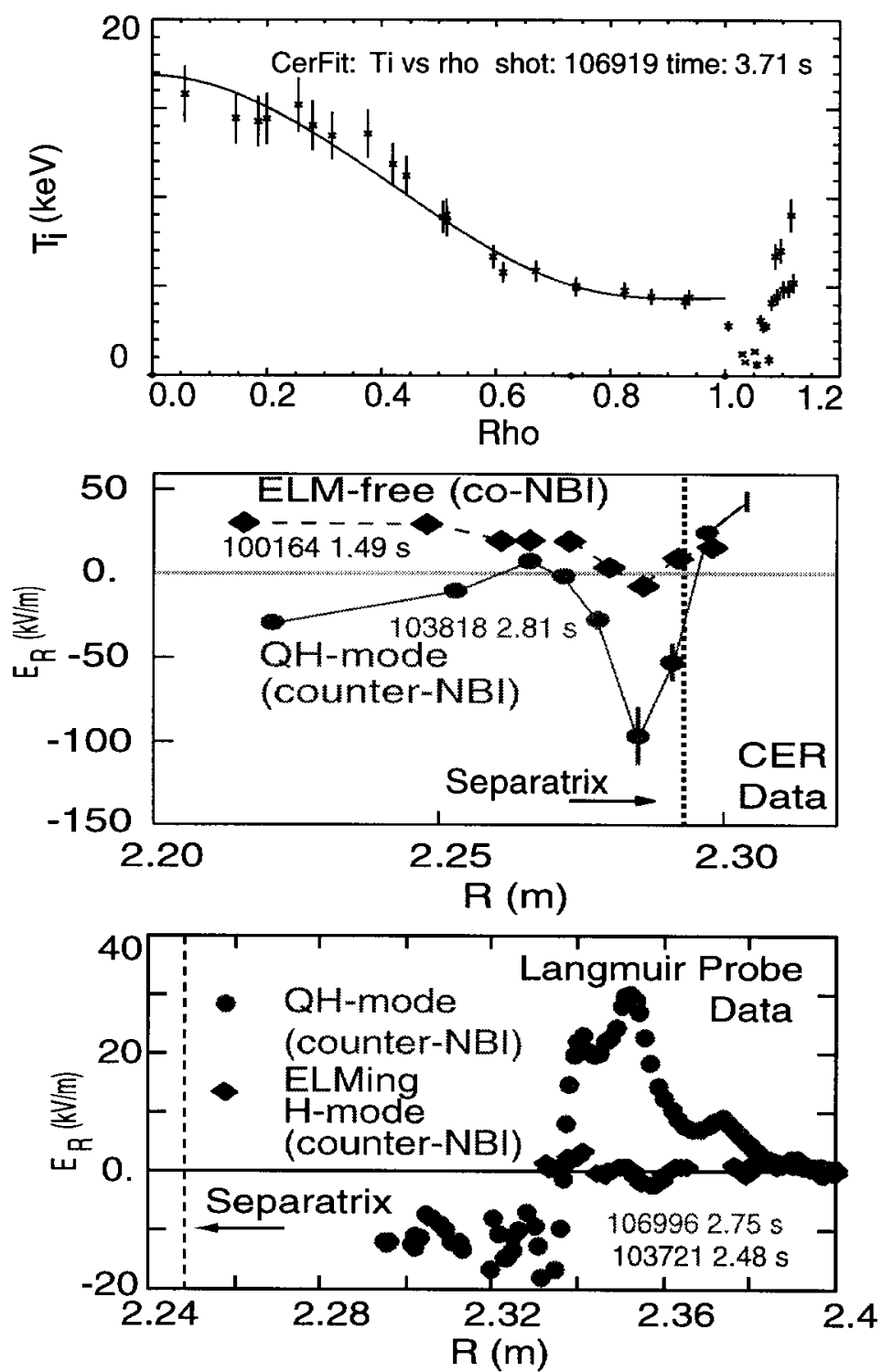

Figure 2 


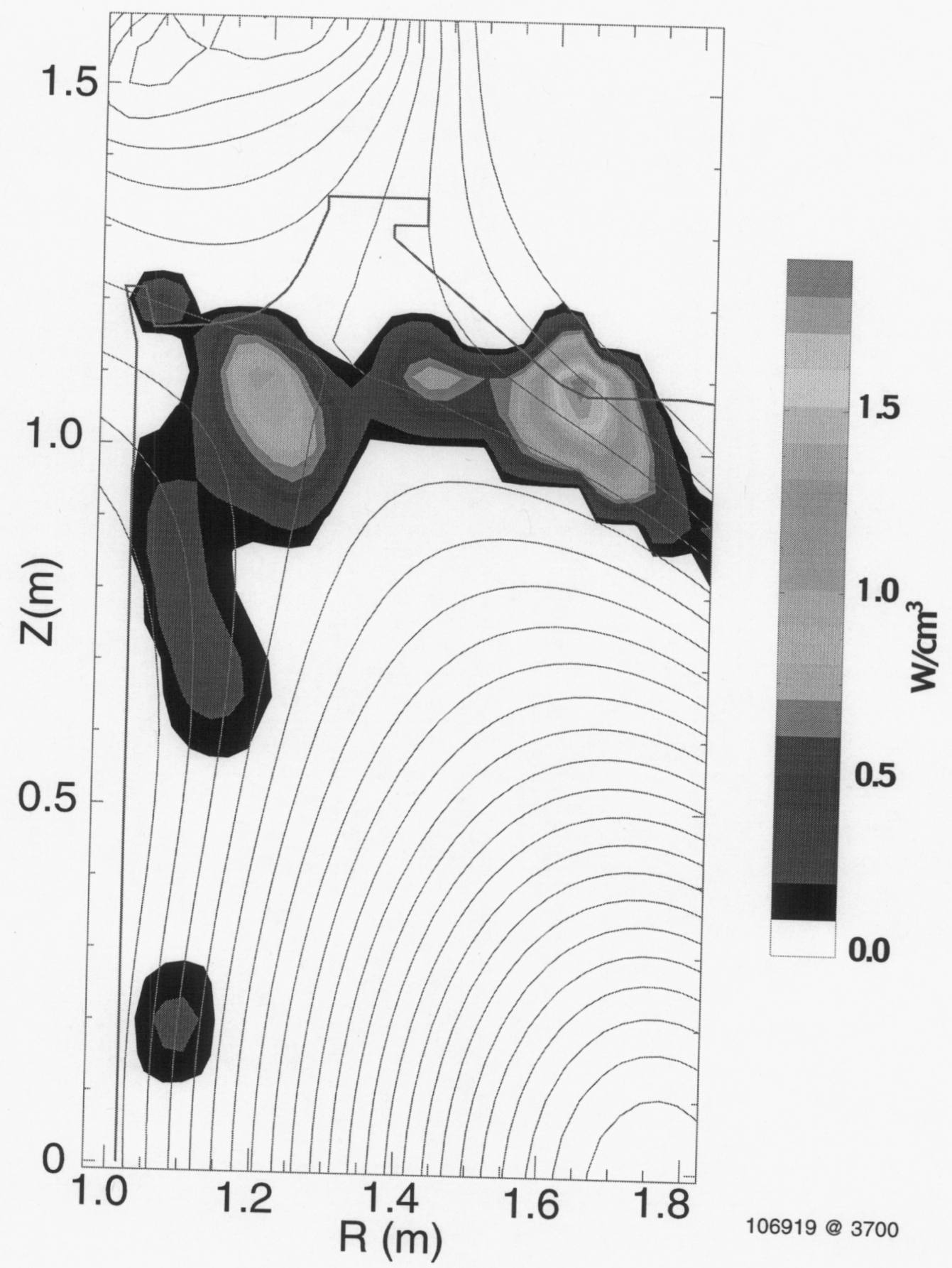

Figure 3 


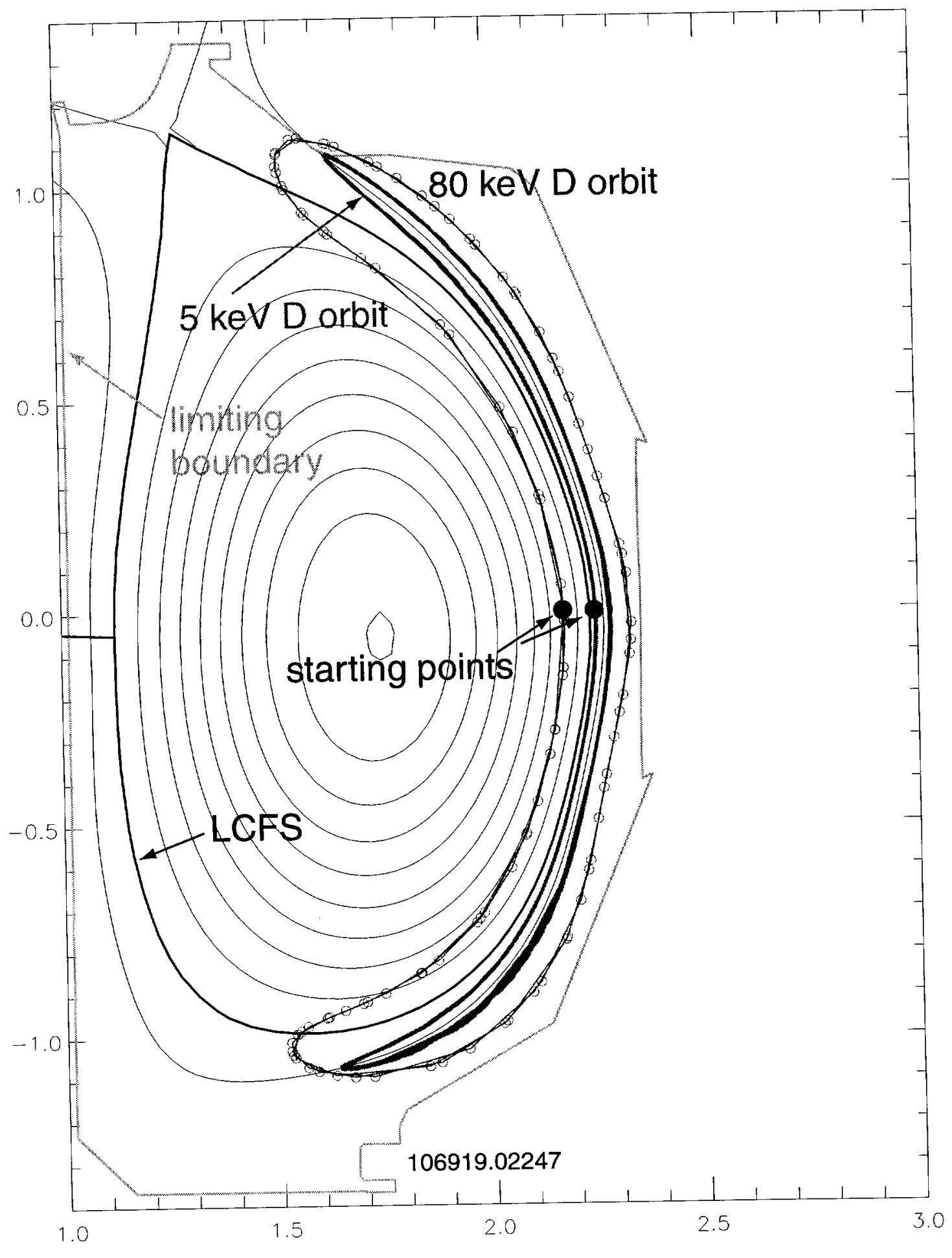

Figure 4 